\title{
Lei dos Processos de Absorção da Radiação
}

\author{
Rogério Custodio \\ Lauro T. Kubota \\ João Carlos de Andrade* \\ dandrade@iqm.unicamp.br \\ Universidade Estadual de Campinas, Instituto de Química
}

\section{Informações do Artigo}

Histórico do Artigo

Criado em Abril de 1999

Atualizado em Março de 2000

\author{
Palavras-Chaves \\ Lei de Beer \\ Transmitância \\ Absorbância \\ Intensidade da radiação \\ Radiação monocromática \\ $\mathrm{UV}-\mathrm{Vis}$ \\ Espectrofotômetro \\ Calibração \\ Concentração
}

\section{Resumo}

A radiação eletromagnética que uma amostra absorve pode revelar algumas de suas características. A Lei de Beer estabelece uma relação linear entre a absorbância e a concentração da amostra, sendo portanto, possível determinar a concentração de uma amostra através de sua curva de calibração (absorbância versus concentração).
Lei dos processos de absorção da radiação

Em estudos quantitativos envolvendo absorção de radiação, necessita-se de uma medida experimental que caracterize a quantidade de radiação eletromagnética absorvida por uma amostra. Esta quantidade corresponde ao que se chama de potência radiante, ou seja, a quantidade de energia característica da radiação por unidade de tempo. A unidade de potência é o Watt e sua magnitude pode variar com a direção. Desta forma, torna-se conveniente definir a potência radiante em função de um plano perpendicular à direção do fluxo da radiação (Figura 1). Experimentalmente, a radiação absorvida por uma amostra é determinada comparando-se a potência radiante do feixe transmitido na ausência de espécies absorventes com a potência radiante transmitida na presença destas espécies.

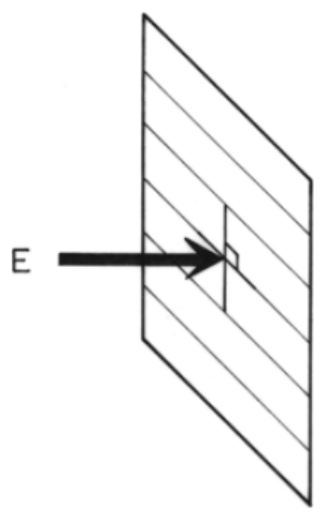

Figura 1 - Feixe de radiação com energia E incidindo perpendicularmente a uma superfície. 
Embora as medidas experimentais sejam realizadas em função da potência radiante, usualmente encontramos na literatura informações sobre a intensidade da radiação. Estas duas quantidades não correspondem à mesma coisa, mas estão relacionadas entre si. A Intensidade de radiação, $I$, é definida como a razão entre a potência radiante e o ângulo sólido1 de incidência. Quando a área iluminada, o ângulo sólido ${ }^{1} \mathrm{e}$ o volume do absorvedor são pequenos, que é o caso das medidas para fins analíticos, a potência da radiação pode ser tomada como a sua intensidade.

Quando um feixe (monocromático ${ }^{2}$ ) de radiação, com intensidade Io, incide sobre uma cubeta contendo uma solução, vários fenômenos podem ocorrer. O efeito mais significativo ocorre quando parte da radiação é absorvida pelo meio que está sendo analisado. Entretanto, este não é o único efeito que pode ser observado. Parte da radiação incidente pode ainda ser refletida, em função do absorvedor ou das diferenças entre o índice de refração do meio onde a radiação se propaga e do meio que está sendo analisado (inclusive pelas paredes da cubeta), enquanto que outra parte poderá ser simplesmente espalhada, caso o meio não seja transparente e homogêneo. Consequentemente, a intensidade do feixe que é medida após a passagem pela amostra (intensidade transmitida, It) será menor que a intensidade inicial, Io. Entretanto, um aspecto extremamente importante, é que todos estes efeitos associados à intensidade de radiação (Figura 2), estão relacionados entre si por uma expressão linear descrita pela equação:

$$
I o=I r+I e+I a+I t \quad \text { equação (1) }
$$

$I o=$ Intensidade do feixe incidente,

$I r=$ Intensidade do feixe refletido, resultado das diferenças do índice de refração entre o absorvedor e o ambiente, $I e=$ Intensidade do feixe espalhado, resultado de um meio não homogêneo (suspensão) e/ou de flutuações térmicas,
$I a=$ Intensidade do feixe absorvido pelo meio $I t=$ Intensidade do feixe transmitido.

Em medidas analíticas convencionais, o efeito de reflexão da radiação eletromagnética, Ir, pode ser minimizado com o uso de medidas relativas e de cubetas com paredes homogêneas de pequena espessura e de faces paralelas. Em outras palavras, preparam-se duas cubetas, uma contendo a amostra que se deseja analisar e outra contendo todos os componentes da cubeta anterior, menos a substância de interesse. A medida da intensidade do feixe transmitido através da segunda cubeta servirá de referência para calibrar o instrumento, antes da medida da intensidade transmitida através da cubeta contendo o material de interesse. Este procedimento é empregado para se descontar os possíveis efeitos de reflexão observados no experimento. Pode-se, desta forma, perceber a necessidade do uso de cubetas praticamente idênticas, com paredes de pequena espessura e com faces paralelas. O procedimento geral para calibração ${ }^{3}$ do espectrofotômetro é de fácil execução.

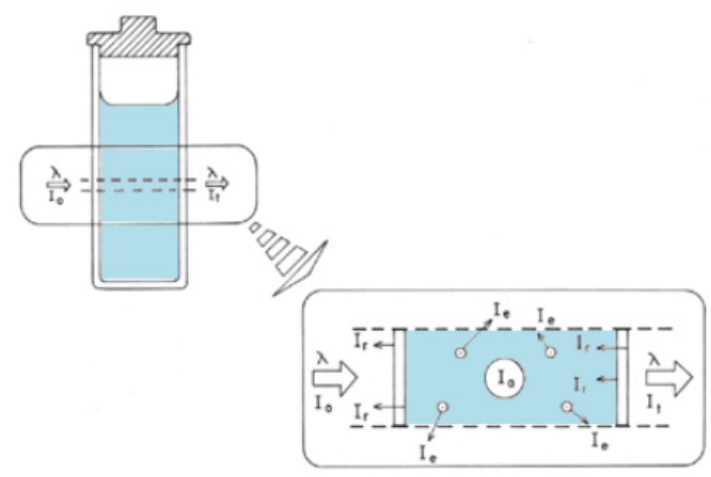

Figura 2 - Fenômenos envolvidos quando um feixe (monocromático) de radiação incide sobre uma cubeta contendo uma solução que absorve no comprimento de onda incidente.

Boa parte dos procedimentos envolvendo absorção de luz é realizada com soluções homogêneas e transparentes, de

\footnotetext{
${ }^{1}$ Um ângulo (q) é definido como a divisão entre o comprimento do arco de uma circunferência (1) pelo raio (r) da mesma: q=1/r. Um ângulo é uma constante adimensional e mede o grau de abertura do arco na superfície da circunferência. Um ângulo sólido (W) é definido como a divisão entre uma área na superfície de uma esfera (A) pelo raio ao quadrado da mesma: $\mathrm{W}=\mathrm{A} / \mathrm{r}^{2}$. Um ângulo sólido também é uma constante adimensional e mede o grau de abertura da área na superfície de uma esfera.

${ }^{2}$ A absorção de radiação e suas leis estão teoricamente relacionados com feixes de radiação monocromáticas (comprimento de onda, 1 , único e definido). Entretanto, as medidas espectrofotométricas usuais estão geralmente associadas a uma banda de radiação (feixe com vários 1). Por isso a palavra "monocromática" será sempre deixada entre parêntesis neste texto.

${ }^{3}$ Procedimentos gerais para a calibração de um espectrofotômetro, para medidas usuais de concentração: Quando são usados aparelhos de feixe simples, o procedimento consiste em ajustar o nível de 100\% de transmitância (zero de absorbância) do equipamento com uma cubeta contendo todos os componentes da solução a ser medida, menos ao substância de interesse ("branco"), e o nível 0\% de transmitância com o obturador do aparelho fechado. As demais medidas serão feitas em relação ao branco, substituindo-o pelas amostras. Para equipamentos de duplo feixe, a radiação proveniente do monocromador é igualmente dividida em dois feixes, que incidem em dois compartimentos, o de referência e o da amostra. O ajuste inicial é feito colocando-se o "branco" nos dois compartimentos e regulando-se o aparelho para absorbância zero, e as leituras são feitas substituindo-se o "branco" do compartimento da amostra pelas amostras a serem medidas. O usuário deverá ler atentamente o manual do seu equipamento para se informar sobre os demais detalhes operacionais. Os termos transmitância e absorbância serão definidos mais ao final deste texto.
} 
modo que a intensidade de radiação espalhada, Ie, pode ser considerada desprezível. Desta forma, trabalhandose com soluções homogêneas, a intensidade da radiação incidente pode ser considerada como sendo utilizada em dois processos, descritos pela equação abaixo:

$$
I o=I a+I t \quad \text { equação (2) }
$$

As intensidades incidentes (Io) e transmitida (It) podem ser medidas diretamente. Logo, a parte absorvida ( $I a)$ pode ser determinada como a diferença entre $I o$ e $I t$.

Algumas técnicas analíticas, tais como a turbidimetria e a nefelometria, utilizam exatamente a propriedade que determinadas soluções não homogêneas apresentam de espalharem luz. Para tais procedimentos, a intensidade do feixe espalhado será o fator determinante para aplicações analíticas. Neste texto, particularmente, não serão discutidos os elementos relacionados com espalhamento de luz, apenas os relacionados às soluções homogêneas.

Quando se pensa em avaliar as propriedades de soluções em função da intensidade de feixes de luz, imagina-se inicialmente que este é o tipo de informação que está intrinsecamente associado a utilização de luz elétrica ou de equipamentos especiais. Entretanto, ao se procurar as origens dos estudos de absorção de luz por soluções, depara-se com a surpresa de que estes estudos são anteriores a descoberta da luz elétrica. Eram utilizadas a luz solar ou a luz emitida por chamas.

Entre as primeiras investigações sobre a relação existente entre as intensidades de radiação incidente e transmitida, destacam-se as experiências de Pierre Bouguer (1729) e de Johann Heindrich Lambert (1760). Estes dois cientistas efetuaram observações independentes e verificaram que as propriedades associadas ao processo de absorção de luz podem ser enunciadas em termos de duas leis fundamentais:

- A intensidade de luz (monocromática) transmitida por um corpo homogêneo é proporcional à intensidade de luz incidente. Isto é: $I t=k I o$.

- A intensidade de luz (monocromática) transmitida decresce exponencialmente com o aumento da espessura da camada do corpo homogêneo.

A Figura 3 mostra claramente a ocorrência deste fenômeno, na rotina do laboratório, com o uso da luz solar.

Esta lei é representada pela equação 3 e pode ser deduzida matematicamente de diversas maneiras:

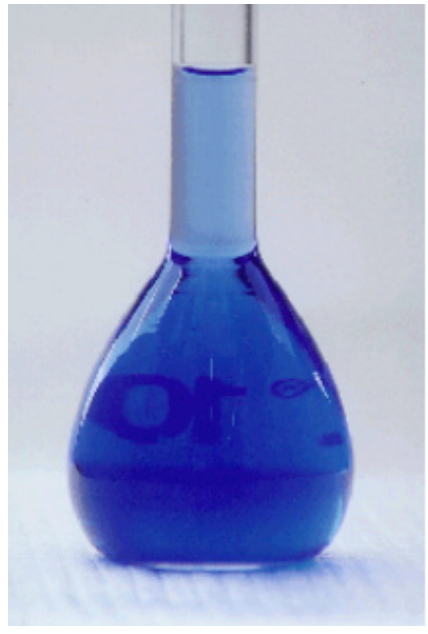

Figura 3 - Solução de $\mathrm{CuSO}_{4}$ em meio amoniacal, contida em um fraco volumétrico. Notar que a solução parece ser mais clara na região de ajuste do menisco, porque o diâmetro do tubo (espessura do absorvedor) é bem menor que na parte inferior do frasco.

$$
I t=I o e^{-k^{\prime} b} \quad \text { equação (3) }
$$

onde: $e$ é a base dos logaritmos naturais e $k$ ' o coeficiente de absorção. Considerando-se a seguinte propriedade matemática associada à potenciação:

$$
\left(a^{b}\right)^{c}=a^{b c}=d^{j c} \quad \text { equação (4) }
$$

onde $a^{b}=d^{b}$, pode-se converter o termo $e^{k}$ em $10^{a}$, convertendo-se a equação 3 em:

$$
I t=I 010^{-a b} \quad \text { equação }(5)
$$

Em 1852, August Beer estudou a influência da concentração de soluções coloridas sobre a transmissão de luz. A conclusão a que se chegou foi de que o valor de para uma determinada substância é proporcional à sua concentração, isto é:

$$
a^{\prime}=a \cdot c
$$

onde, c é a concentração e é a absortividade (constante independente da concentração).

A lei de Beer é análoga à lei de Bouguer - Lambert. Enquanto Bouguer e Lambert estudaram a variação na absorção de um feixe de luz, em função da variação da espessura da camada absorvente, Beer fez o mesmo estudo no que se refere à concentração da solução, mantendo a espessura constante. Em ambos os casos, o resultado é o mesmo pois, quer se varie a concentração, quer se varie a espessura da solução a ser atravessada pela luz, em essência, aumentamos ou diminuímos o número de partículas que interagem com a radiação.

Combinando-se as equações 4 e 5 , obtém-se a lei básica 
da espectrofotometria, ou seja a Lei Bouguer - Lambert - Beer, mais conhecida como Lei de Beer:

$$
I t=I 0 e^{-a c b} \quad \text { equação (6) }
$$

Se a concentração $\mathrm{c}$ for expressa em mol por litro e a espessura do absorvedor (neste caso o caminho óptico da cubeta, b) em centímetros, a constante toma o nome de absortividade molar, e o símbolo normalmente empregado para esta quantidade passa a ser $\varepsilon$ (épsilon). A absortividade, a, é a mais usada quando não se conhece a natureza do material absorvente (e portanto a sua massa molar), sendo a concentração expressa em gramas por litro. A absortividade molar ( $\varepsilon$ ) é preferível quando se deseja comparar quantitativamente a absorção de várias substâncias. Para uma mesma espessura do absorvedor (caminho óptico), quanto maior o valor de $\varepsilon$ maior a sensibilidade do método. Assim sendo, pode-se escrever a equação 6 como:

$$
I t=I 0 e^{-z c b} \quad \text { equação }(7)
$$

A absortividade molar, $\varepsilon$, depende da substância, do comprimento de onda utilizado, da temperatura e do solvente. Analisando-se a equação 7, percebe-se que quanto maior o valor de $\varepsilon$, maior será a taxa de absorção observada e mais sensível o método espectrofotométrico. Esta é a razão pela qual, idealmente, procura-se trabalhar com uma radiação monocromática, sempre que possível, correspondente ao máximo de absorção da espécie a ser determinada.

Rearranjando-se a equação 7 obtém-se:

$$
I t / I o=10^{-z c b} \quad \text { equação (8) }
$$

A relação It / Io chama-se transmitância e é representada pelo símbolo T. Logo:

$$
T=10^{-z c b} \quad \text { equação (9) }
$$

Percebe-se pela equação 9 que a relação entre $\mathrm{T}$ e c não é linear, o que tende a dificultar a comparação entre diferentes transmitâncias e as concentrações a elas associadas. No entanto, aplicando logaritmos à equação 8 (ou 9), obtém-se:

$$
\log _{10} I 0 / I t=-\log _{10} T=\varepsilon c b \quad \text { equação (10) }
$$

A relação $\log _{10}$ (Io / It) é chamada de absorvância por muitos autores de língua portuguesa, mas diversos outros preferem a derivação do inglês, absorbância. Por questões de uso, este texto utilizará esta última alternativa. Assim, a Lei de Beer pode ser representada como na Figura $4 \mathrm{e}$ enunciada simplesmente como:
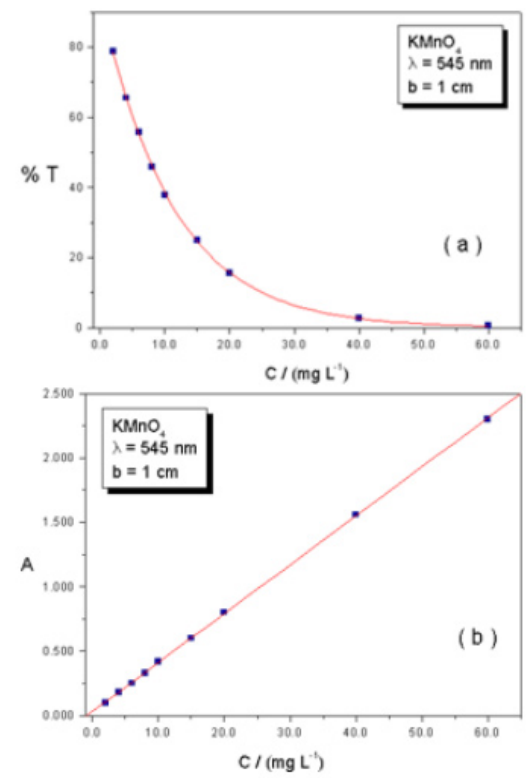

Figura 4 - Representação gráfica da Lei de Beer, para soluções de $\mathrm{KMnO}_{4}$ em $\lambda=545 \mathrm{~nm}$ e um caminho óptico de $1 \mathrm{~cm}$. a) $\mathrm{Em}$ \%Transmitância [\%T versus c - eq. 9]; b) Em Absorbância [A versus c - eq. 12].

$$
A=\varepsilon b c \quad \text { equação (11) }
$$

ou no caso de não se utilizar a concentração em mol por litro:

$$
A=a b c \quad \text { equação (12) }
$$

Percebe-se claramente, na equação 12 , a relação linear entre a absorbância, A, e a concentração c. Portanto, mantendo-se o caminho óptico constante, pode-se determinar a concentração de uma espécie em solução, através da medida de absorbância. Na prática, uma curva de calibração (absorbância versus concentração), da espécie de interesse é construída e a concentração da amostra é determinada através dela.

Para se realizar uma análise espectrofotométrica ainda é necessário conhecer o espectro de absorção da amostra que se quer determinar. Isto é feito para se definir qual o comprimento de onda da radiação incidente que causará o máximo de absorção pela espécie a ser determinada e assim obter-se a melhor sensibilidade na sua quantificação. O espectro de absorção é obtido variando-se o comprimento de onda da radiação que incide sobre a amostra e medindo-se a quantidade de radiação absorvida em um espectrofotômetro.

Uma outra característica particular da lei de Beer é a 
aditividade das absorbâncias. Em muitos casos, é ainda possível determinar simultaneamente duas ou mais espécies diferentes presentes numa amostra, utilizando esta mesma lei. Teoricamente, isto pode ser realizado desde que não ocorra nenhuma interação entre as espécies e que o espectro de absorção observado pela mistura seja a soma dos espectros individuais que seriam obtidos caso cada uma das espécies estivesse presente sozinha na solução e sob as mesmas condições experimentais. Na prática, estas condições ideais não ocorrem mas, mesmo assim, ainda é possível a determinação de espécies químicas em uma mistura. Neste caso, para cada comprimento de onda, a absorbância total devido às espécies presentes na solução pode ser expressa como a soma das absorbâncias de cada uma delas,

$$
A_{\lambda i}=A_{\lambda n}=b \sum \varepsilon_{n} c_{n}
$$

Para o caso particular de duas substâncias,

$$
\begin{aligned}
& A_{\lambda 1}=A_{1, \lambda 1}+A_{2, \lambda 1} \\
& A_{\lambda 2}=A_{1, \lambda 2+} A_{2, \lambda 2}
\end{aligned}
$$

onde $A_{\lambda 1}$ e $A_{\lambda 2}$ são os valores de absorbância medidas em dois comprimentos de onda diferentes, $\lambda 1$ e $\lambda 2$, e os índices 1 e 2 representam as duas substâncias diferentes.

\section{Referências Bibliográficas}

1. Strong, F.C., "Theoretical basis of the Bouguer-Beer law of radiation absorption”, J. Chem. Educ., 1952, 24: 338-347 [com correções de algumas equações em J. Chem. Educ., 1952, 24: 2013].

2. Swinehart, D.F., "The Beer-Lambert law", J. Chem. Educ., 1962, 39: 333-335.

3. Barberan-Santos, M.N., "The Beer law revisited", J. Chem. Educ., 1990, 67: 757-759 [ver também as citações bibliográficas deste artigo].

4. Lykos, P., "The Beer-Lambert law revisited - a development without calculus", J. Chem. Educ., 1992, 69: 730-732. 